\title{
A role for the seminal vesicles in sex drive and sexual activity in mice
}

Although their role in fertility is well known, data supporting suggestions that the seminal vesicles might be involved in sexual activity and sex drive have so far proved elusive. Now a study published in European Urology has investigated this hypothesis in a mouse model, producing data to support such a role for the seminal vesicles.

Male mice were randomized to one of three different groups-bilateral seminal vesicle occlusion (SVO), in which the vesicles were occluded using titanium

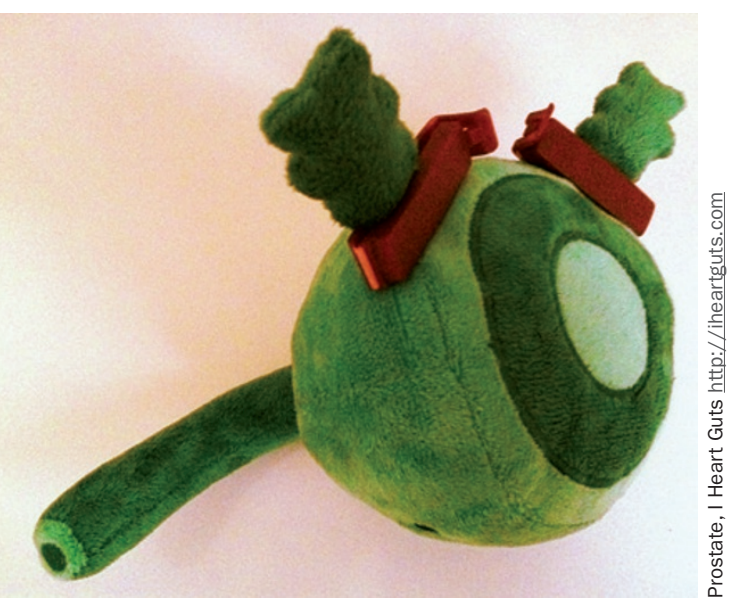

clips, bilateral seminal vesicle resection (SVR) or sham operation (SO).

10-14 days after surgery, the male mice were placed near to cages of females in order to expose the females to male hormones for 3 days, which induces and synchronizes oestrus in the females-a phenomenon known as the Whitten effect. Male mice were then exposed to a female in controlled conditions during the dark phase of the day/night cycle and sexual activity was observed for sessions of $3 \mathrm{~h}$. After all observation sessions had been completed, the mice were sacrificed and the status of the seminal vesicles assessed.

A total of $423 \mathrm{~h}$ of observation time was completed by 77 male mice, although perioperative mortality meant that the SVO and SVR groups were smaller than the SO group. The primary end point of the study was the number of sessions in which intromission occurred for each group of mice. In the SVO males, intromission occurred in a total of 20 of 42 sessions (48\%), compared with only eight of 39 sessions (21\%) and 18 of 60 sessions (30\%) in SVR and SO males, respectively. Secondary end points studied—number of intromissions and latency time before intromission per session-did not vary between the three groups. Necropsy confirmed that the seminal vesicles in the SVO group of mice were substantially enlarged compared with those of the SO group $(2.5-3 \mathrm{~cm}$ versus $1.5 \mathrm{~cm})$.

These data support a role for seminal vesicles in sexual function with occlusion and, therefore, engorgement of the seminal vesicles increasing the level of sexual activity. These observations led the authors to conclude that these organs have an intrinsic role in stimulating sexual activity.

The data are supported by reports that men who have undergone seminalvesicle-sparing prostatectomy maintain a higher sex drive than those who have had their seminal vesicles removed. These data could provide an added incentive for sparing the seminal vesicles during prostate surgery.

\section{Annette Fenner}

Original article Birkhäuser, F. D. et al. Occlusion of seminal vesicles increases sexual activity in a mouse model. Eur. Urol. doi:10.1016/j.eururo.2012.04.036 